\title{
Caenorhabditis elegans susceptibility to gut Enterococcus faecalis infection is associated with fat metabolism and epithelial junction integrity
}

\author{
Shuzhen $\operatorname{Sim}^{1 *}$ (D) and Martin L. Hibberd ${ }^{1,2}$
}

\begin{abstract}
Background: Gut bacteria-host interactions have been implicated in the pathogenesis of numerous human diseases, but few mechanisms have been described. The genetically tractable nematode worm Caenorhabditis elegans can be infected with pathogenic bacteria, such as the human gut commensal Enterococcus faecalis, via feeding, making it a good model for studying these interactions.

Results: An RNAi screen of 17 worm candidate genes revealed that knockdown of the transcription factor nhr-49, a master regulator of fat metabolism, shortens worm lifespan upon infection with E. faecalis (and other potentially pathogenic bacteria) compared to Escherichia coli. The functional similarity of nhr-49 to the mammalian peroxisome proliferator-activated receptors (PPARs) suggests that this is mediated through a link between fatty acid metabolism and innate immunity. In addition, knockdown of either dlg-1 or ajm-1, which encode physically interacting proteins in the C. elegans epithelial junction, also reduces worm lifespan upon E. faecalis challenge, demonstrating the importance of the intestinal epithelium as an immune barrier.

Conclusions: The protective roles identified for $n h r-49$, dlg-1, and ajm-1 suggest mechanistic interactions between the gut microbiota, host fatty acid metabolism, innate immunity, and epithelial junction integrity that are remarkably similar to those implicated in human metabolic and inflammatory diseases.
\end{abstract}

Keywords: Gut microbiome, Host-microbe interactions, Nuclear receptors, Fat metabolism, Epithelial junction integrity

\section{Background}

The human gastrointestinal tract harbors an enormous number and variety of microbes, with a collective genome size estimated at 150 times the complement of human genes [1]. These organisms, collectively termed the gut microbiome, carry out crucial metabolic and biological functions. They aid in food digestion and nutrient uptake, play important roles in the development of the immune system and intestinal epithelial barrier, and compete with potentially pathogenic microorganisms, thus curbing their growth (reviewed in [2,3]). Studies of germ-free or gnotobiotic mice have shown that these

\footnotetext{
*Correspondence: shuzhens@gis.a-star.edu.sg

${ }^{1}$ Infectious Diseases, Genome Institute of Singapore, 60 Biopolis Street, \#02-01 Genome, Singapore 138672, Singapore

Full list of author information is available at the end of the article
}

microbes also have local and systemic effects outside of the gut [4-8], highlighting the complex nature of their interactions with the host, although the mechanisms of these interactions require further research.

Disturbances of the gut microbiome through factors such as diet, antibiotic treatment, or pathogen invasion can lead to dysbiosis, in which there is an outgrowth of potentially pathogenic bacteria and a decrease in beneficial bacteria. The consequent alteration in host interactions is thought to result in a perturbed immune balance, which has been linked to the pathogenesis of diverse intestinal and systemic human diseases (reviewed in [9-11]). These include inflammatory bowel diseases (IBD) such as ulcerative colitis and Crohn's disease [12-15], and metabolic disorders or conditions such as metabolic syndrome, obesity, and type 2 diabetes (T2D) [16-19]. 
Further evidence comes from the perspective of human genetics (reviewed in [20]). Genome-wide association studies (GWAS) have identified loci associated with susceptibility to IBD [21-23] and T2D [24, 25] that are also associated with microbial defense, thus supporting a role for host-microbiome interactions in disease pathogenesis. For example, NOD2, the first gene to be associated with IBD, encodes an intracellular pattern recognition receptor that mediates the host immune response to the bacterial peptidoglycan product muramyl dipeptide (MDP) [26]; other IBD-associated genes with roles in anti-bacterial immunity include CARD9, IL23R, and LRRK2 [21-23]. Interestingly, there is considerable overlap between risk loci for Crohn's disease and leprosy [27-29], suggesting a potential mycobacterial component to the pathogenesis of IBD. However, studies that have reported links between microbial dysbiosis, host genetics, and disease often lack direct causal or functional evidence.

To address this lack of functional evidence, we have utilized the genetically tractable nematode worm Caenorhabditis elegans, a commonly used model for understanding microbial host-pathogen interactions [30-33]. Since C. elegans is a bacterivore, intestinal infection can be achieved through feeding, making it especially ideal for investigating interactions with gut bacteria. In this study, to identify potentially pathogenic host-microbiome interactions, we selected nematode candidate genes whose orthologs or functionally related genes in the human have previously been implicated in metabolic or inflammatory diseases. An RNAi screen of these genes identified two aspects of nematode biology - fatty acid metabolism and epithelial junction integrity-that are involved in C. elegans defense against the human gut commensal Enterococcus faecalis, an opportunistic pathogen commonly associated with hospital-acquired, multidrug-resistant infections [34].

\section{Results \\ Knockdown of nhr-49 or dlg-1 reduces nematode lifespan upon OG1RF challenge}

OG1RF, an E. faecalis strain of human origin, kills C. elegans by establishing a persistent infection in the nematode intestine, where it proliferates extensively from a small initial inoculum and causes gross distention [31]. The thick cell wall of E. faecalis is thought to protect it against the C. elegans grinder organ, which normally disrupts ingested bacteria [31].

To identify host-bacteria interactions during $E$. faecalis infection of C. elegans, we performed RNAi knockdowns of 17 candidate C. elegans genes (Additional file 1: Table S1), screening for impact on nematode survival upon OG1RF challenge. These candidates have functional domains that suggest potential interactions with bacteria, but had not previously been characterized in the context of microbial infection in the nematode. In addition, their human orthologs or functionally related genes have been implicated in metabolic and inflammatory diseases.

Knockdown of two candidate genes-nhr-49 and $d l g$ 1 -resulted in a significant decrease in nematode lifespan upon exposure to OG1RF, compared to the RNAi vector control (Fig. 1a). As has been previously reported [35], knockdown of $n h r-49$ on OP50 also resulted in a significant decrease in lifespan (Fig. 1b). However, this reduction (from a median of 10 days to 7 days) was not as severe as compared to knockdown on OG1RF (from undefined, i.e. end-point survival exceeded $50 \%$, to 6 days) $(\mathrm{p}<0.0001)$ (Fig. 1b). Knockdown of $d l g-1$ on OP50 had no effect on lifespan (Fig. 1c).

\section{nhr-49 and dlg-1 impact C. elegans interactions with other bacteria}

We also examined the effect of $n h r-49$ and $d l g-1$ knockdown on nematode lifespan upon challenge with other potentially pathogenic bacteria species. Streptococcus gallolyticus gallolyticus (previously classified as $S$. bovis) is a Gram-positive opportunistic human pathogen that has consistently been associated with colorectal cancer, although a direct causal link has yet to be established [36, 37]. Knockdown of $n h r-49$ or $d l g-1$ on SGG53, a clinical S. gallolyticus gallolyticus isolate, also resulted in a significant reduction in nematode lifespan (Fig. 2a and b).

The non-pathogenic, fast-growing Mycobacterium smegmatis strain mc(2) 155 is often used as a laboratory model of mycobacterial disease. GWAS have reported an overlap between susceptibility genes for leprosy (caused by $\mathrm{Myco}$ bacterium leprae) and Crohn's disease [27-29]; Mycobacterium avium paratuberculosis has also been linked to Crohn's (reviewed in [38]). While these studies hint at a potential mycobacterial component to the pathogenesis of IBD, this has not been substantiated with direct evidence. Here, knockdown of $n h r-49$ on mc(2) 155 also significantly reduced nematode lifespan (Fig. 2c), whereas $d l g-1$ knockdown on mc(2)155 showed no effect (Fig. 2d).

Because median lifespans for nematodes maintained on SGG53 and mc(2)155 were undefined, we could not tell if the lifespan reduction seen upon $n h r-49$ knockdown on these bacteria was more or less severe than for knockdown on OP50 (Fig. 2a and c).

Taken together, the data suggest that $n h r-49$ affects interactions with OG1RF, SGG53, and mc(2)155, with the greatest impact on OG1RF, while $d l g-1$ affects interactions with OG1RF and SGG53.

\section{Knockdown of nhr-49 transcriptional targets does not impact survival on OG1RF}

$n h r-49$ encodes a nuclear hormone receptor master regulator of fat metabolism. Loss of $n h r-49$ increases fat content and reduces lifespan in nematodes maintained on OP50 [35]. nhr-49 enhances expression of acs-2, a 

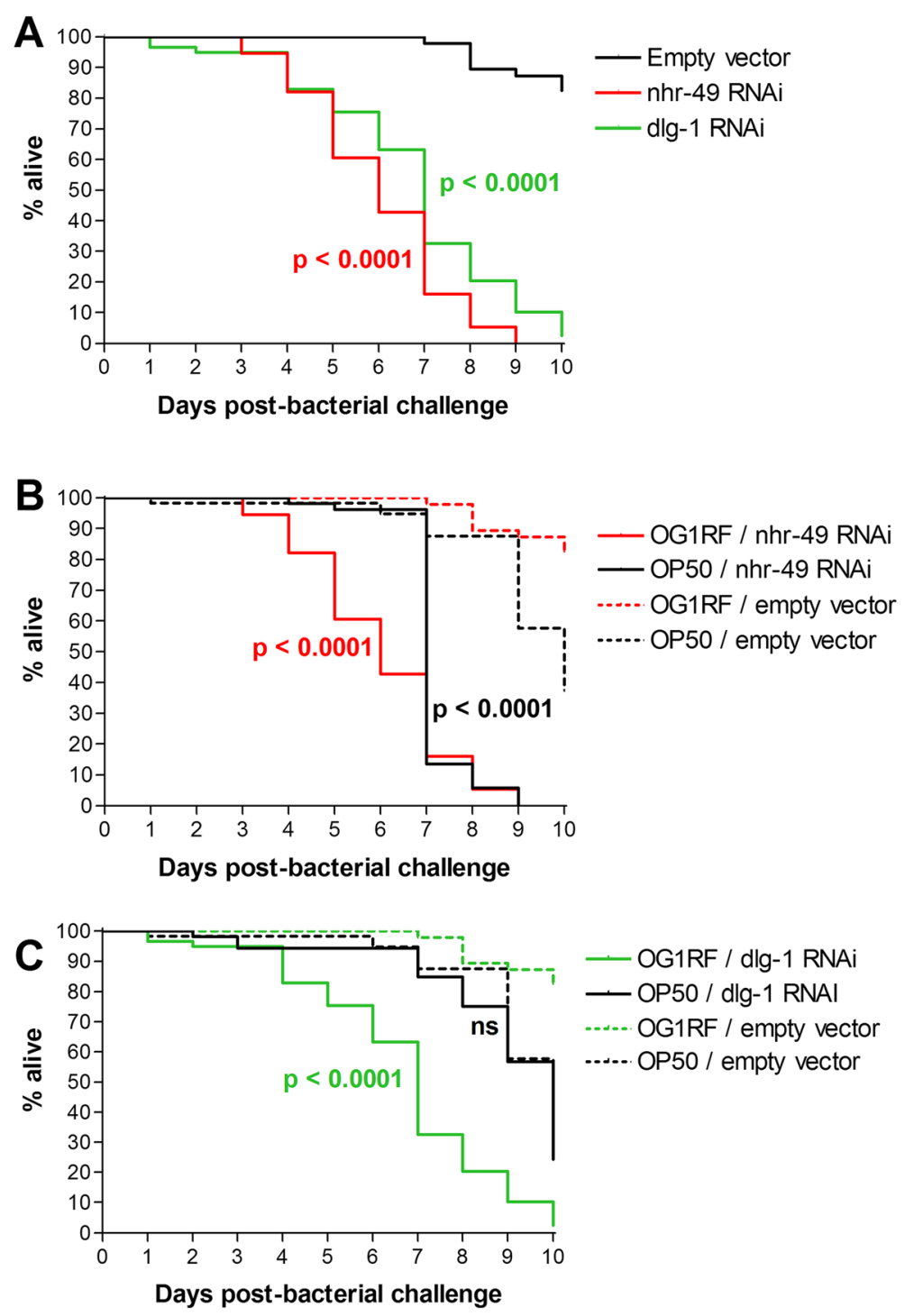

Fig. 1 Effect of gene knockdown on C. elegans lifespan upon challenge with OG1RF. a Kaplan-Meier survival curves showing the effect of nhr-49 and dlg-1 knockdown upon challenge with OG1RF. b-c Data from (a) shown alongside survival curves for nhr-49 and dlg-1 knockdown upon challenge with OP50; OG1RF and OP50 survival assays were performed on separate occasions. Data represent three biological replicates with 20-30 nematodes each; $p$-values indicate comparisons between gene knockdown and empty vector for the same bacteria

mitochondrial acyl-CoA synthetase, and ech-1, a mitochondrial $\beta$-oxidation trifunctional enzyme, thus promoting the flow of fatty acids into the mitochondria for use in energy production. $n h r-49$ also enhances expression of fat-7, a stearoyl-CoA desaturase, thus maintaining proper balance of saturated to unsaturated fatty acids. fat -7 is involved in a negative feedback mechanism that inhibits acs-2 and ech-1 expression, possibly by signaling through an as-yet-unidentified fatty acid species [35] (Fig. 3a).

We examined the effect of OG1RF challenge on $n h r-$ 49, acs-2, ech-1, and fat-7 expression by quantitative RTPCR. By 7 days post-OG1RF challenge, the transcript abundance of acs-2 was induced $\sim 200$-fold $(p=0.0004)$ relative to nematodes maintained on OP50 (Fig. 4a).

We next determined if OG1RF induction of acs-2 was dependent on $n h r-49$ or fat-7. Knockdown of $n h r-$ 49 resulted in a moderately stronger induction of acs-2 upon OG1RF challenge (222-, 163-, and 56-fold versus 54-, 8, and 33-fold relative to worms on OP50) (Fig. 4b). Knockdown of fat 7 resulted in a much more appreciable and consistent induction of acs-2 (178-, 368-, and 916-fold versus 54-, 8-, and 33-fold relative to worms on OP50) (Fig. 4b). However, due to the large range of the data, a student's t-test did not find these changes statistically significant. These data underscore 


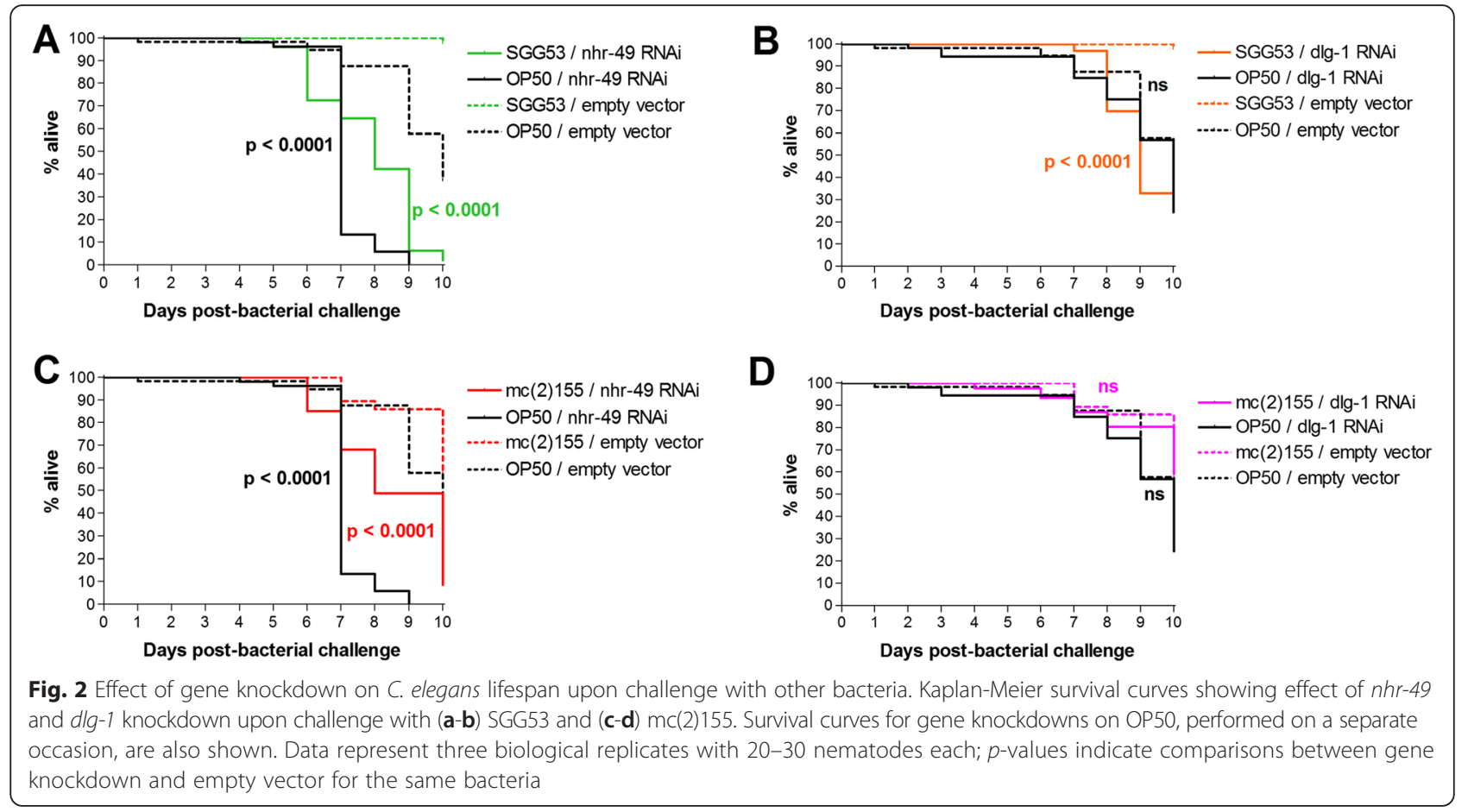

the role of $f a t-7$ as a negative regulator of acs-2, and suggest that $n h r-49$ depletion and subsequent reduced fat -7 transcription may result in moderate loss of this regulation. The relative balance between $n h r-49$ and fat-7 levels are likely to contribute to the variability in the data.

Despite its strong induction by OG1RF, knockdown of acs-2 had no effect on nematode lifespan after bacterial challenge. Similarly, knockdown of either fat-7 or ech-1 separately did not impact lifespan after bacterial challenge (Fig. 5a).

\section{Knockdown of the dlg-1-interacting gene ajm-1 shortens lifespan on OG1RF}

$d l g-1$ encodes a protein that physically interacts with AJM1 in the C. elegans epithelial junction. The DLG-1 / AJM-1 complex is targeted to apical junctions by LET-413 (Fig. 3b) (reviewed in [39]).
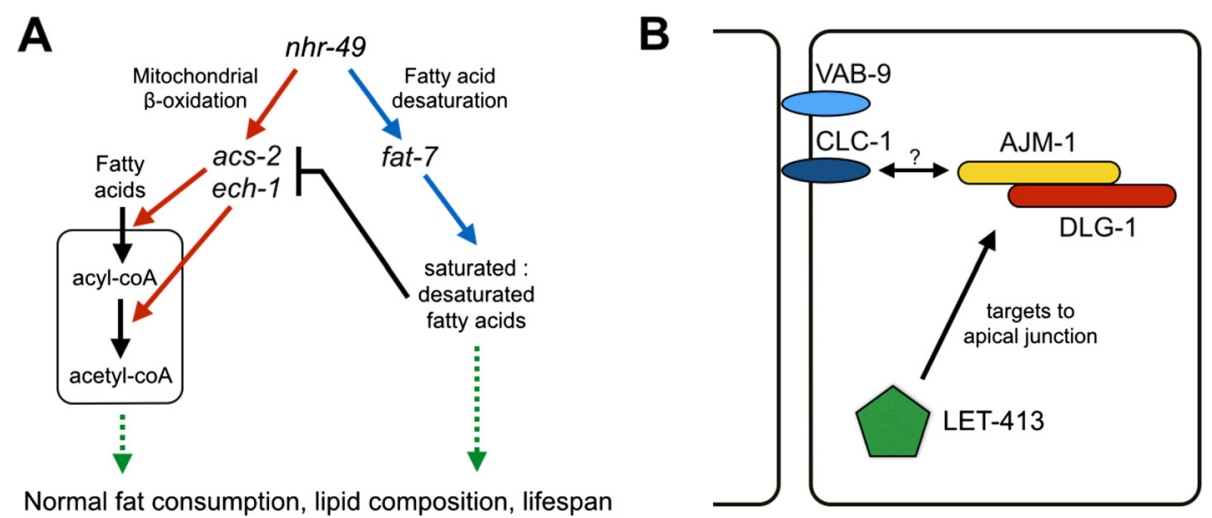

Fig. 3 Modes of action of $n h r-49$ and dlg-1. a nhr-49 encodes a nuclear hormone receptor that enhances the expression of acs-2 and ech-1, facilitating the flow of fatty acids into the mitochondrial matrix. nhr-49 also maintains a normal balance of saturated and unsaturated fatty acids by enhancing expression of fat-7. Deletion or depletion of nhr-49 increases fat content and reduces lifespan in C. elegans maintained on OP50. fat-7 is involved in a negative feedback mechanism that inhibits acs-2 and ech-1 expression, possibly by signaling through an as-yet unidentified fatty acid species. $\mathbf{b} \mathrm{dlg}-1$ encodes a protein that physically interacts with ajm-1 in the C. elegans epithelial junction. The DLG-1 / AJM-1 complex is targeted to apical junctions by LET-413, and may be linked to the cell membrane by claudin-like transmembrane proteins such as VAB-9 or CLC-1. See text for more details; figures have been adapted from [35, 39] 

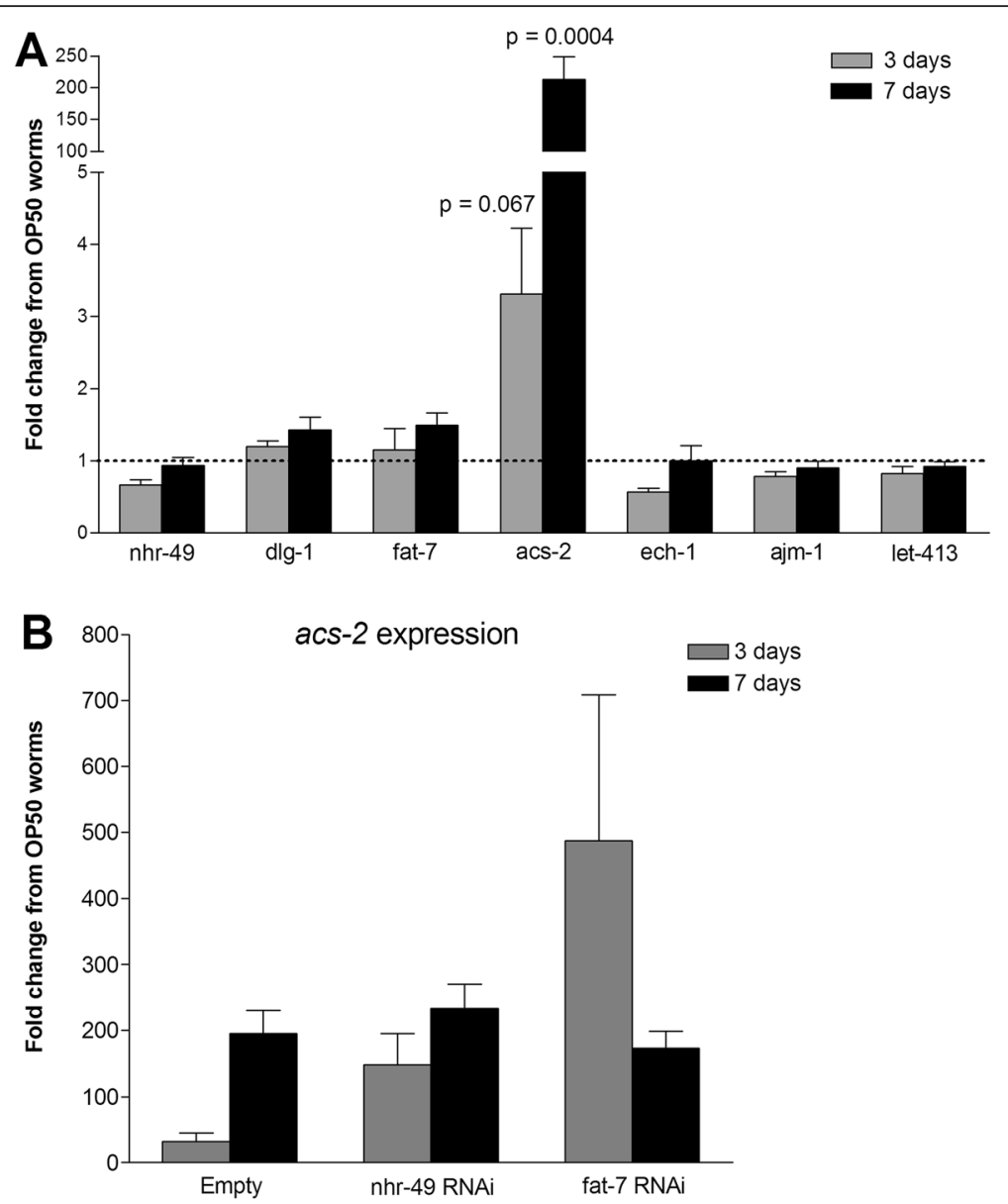

Fig. 4 Effect of OGIRF challenge on C. elegans gene expression. a Fold change in gene expression at 3 and 7 days post-OG1RF challenge, relative to nematodes grown on OP50. b Fold change in acs-2 expression upon gene knockdown, at 3 and 7 days post-OG1RF challenge, relative to gene knockdown on OP50

OG1RF infection did not modulate dlg-1, ajm-1, or let413 expression (Fig. 4a). Knockdown of ajm-1 resulted in a significant decrease in lifespan for nematodes maintained on OG1RF (Fig. 5a) but not on OP50 (Fig. 5b); knockdown of let-413 did not impact lifespan on OG1RF (Fig. 5a).

\section{Discussion}

Insights from human GWAS and studies of germ-free animals suggest that host-microbiome interactions may play roles in triggering inflammatory and metabolic diseases (reviewed in [11, 20]). However, as causative organisms and disease mechanisms have yet to be definitively identified, this concept remains largely speculative. To address this lack of functional evidence, we used the $C$. elegans model system to identify potentially pathogenic host-microbe interactions. We identified two aspects of host physiology_fat metabolism and epithelial junction integrity-that $E$. faecalis interacts with during the killing of C. elegans.
Depletion of $n h r-49$, which encodes a nuclear hormone receptor and master regulator of fat metabolism, shortens worm lifespan upon E. faecalis infection. $n h r-49$ shares strong sequence similarity with the mammalian hepatocyte nuclear factor 4 (HNF4) receptors; mutations in HNF-4A are associated with maturity onset diabetes of the young 1 (MODY1) and T2D (reviewed in [40]). However, $n h r-49$ 's effects on fat metabolism and lifespan are more closely analogous to those of the mammalian peroxisome proliferator-activated receptors (PPARs) [35]. PPARs-nuclear hormone receptor transcription factors activated by fatty acids and their derivatives-are key regulators of metabolism and controllers of inflammation (reviewed in [41]), and are promising targets for the treatment of metabolic syndrome and IBD [42-44].

Obesity, insulin resistance, and other metabolic syndrome-related conditions are characterized by chronic, low-grade inflammation in metabolic tissues such as adipose tissue, liver, skeletal muscle, and blood vessel walls $[11,41]$. Studies in mice have found that gut bacteria- 

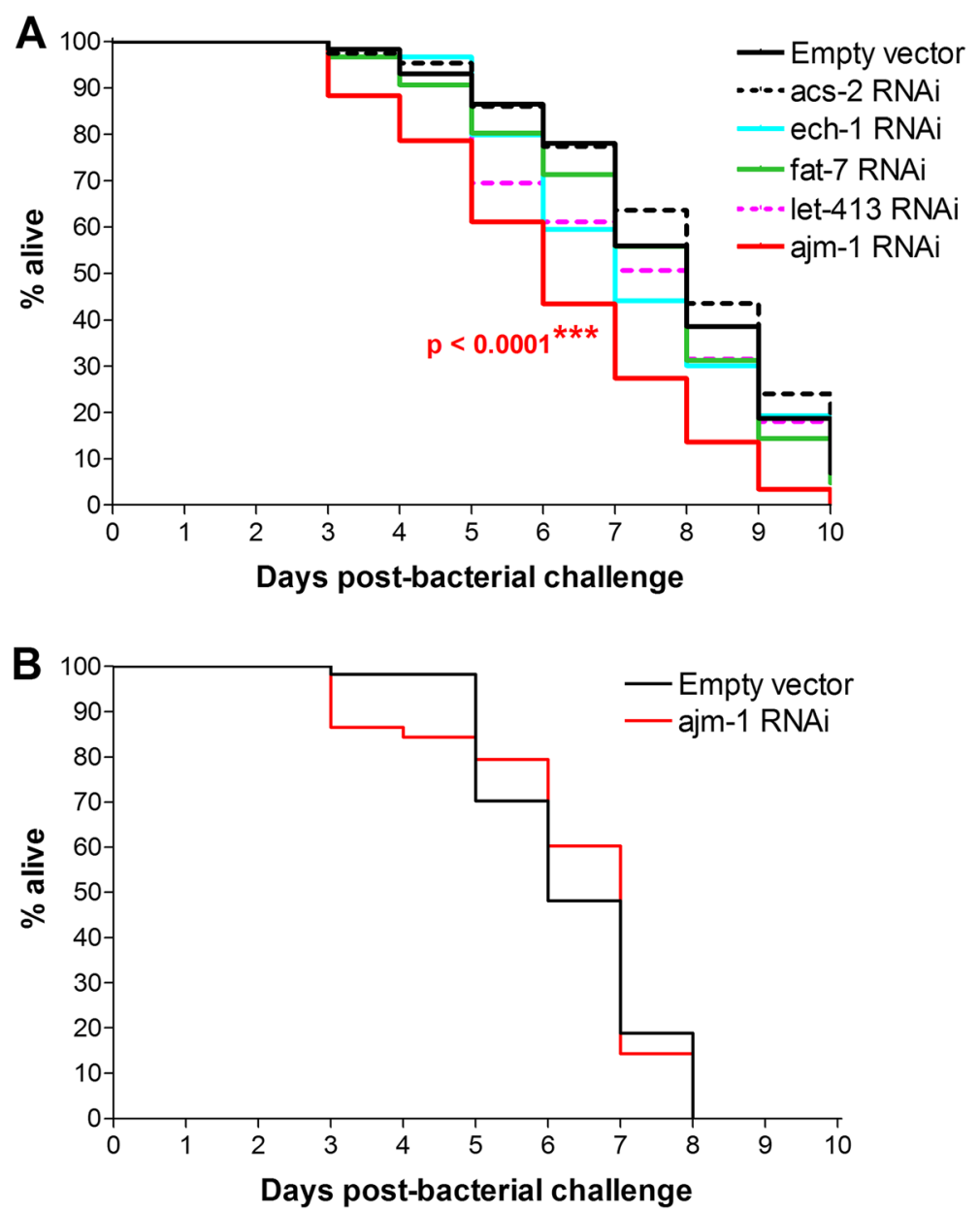

Fig. 5 Effect of knockdown of nhr-49- and dlg-1-interacting genes on C. elegans lifespan upon challenge with OG1RF. a Kaplan-Meier survival curves showing effect of gene knockdown upon challenge with OG1RF. b Kaplan-Meier survival curves showing effect of ajm-1 knockdown on OP50

derived lipopolysaccharide (LPS) can initiate this inflammatory state, and consequently obesity and insulin resistance, by binding to CD14-TLR4 complexes on immune cells $[45,46]$. High fat diets can cause changes in the gut microbiota composition that result in increased circulating LPS levels [45].

PPARs control inflammation through multiple mechanisms (reviewed in [41]). In adipose tissue and the liver, the presence of alternatively activated macrophages (AAMs), which dampen inflammation and improve insulin sensitivity, is maintained by PPAR-dependent mechanisms $[47,48]$. PPARs also reduce inflammation in muscle cells by regulating saturated fatty acid metabolism away from diacylglycerol accumulation, thus avoiding NF- $\mathrm{kB}$ activation [49].

PPARs also regulate inflammation in the intestinal epithelium, and there is ample evidence for more direct, local interactions between these molecules and gut bacteria. For example, the human gut commensal Bacteroides thetaiotaomicron enhances complex formation between PPAR $\gamma$ and the NF- $\mathrm{kB}$ subunit RelA in human intestinal cell lines, resulting in PPAR $\gamma$-dependent nuclear export of RelA and subsequent suppression of proinflammatory cytokine production [50]. In addition, $E$. faecalis isolated from newborn babies induces phosphorylation and activation of PPAR $\gamma$ in colonic epithelial cell lines and primary colonic cells, resulting in transcriptional activation of target genes, including the immunemodulatory cytokine IL-10 [51]. These studies suggest potential links between fatty acid signaling / metabolism and innate immunity [41], and, similar to what we observe here for $n h r-49$, a protective role for PPARY in gut homeostasis.

In our study, E. faecalis infection strongly induced expression of the $n h r-49$ transcriptional target acs-2, an acyl-CoA synthetase that activates fatty acids for $\beta$ oxidation and energy production in the mitochondria. Both pathogenic and commensal bacteria, including $E$. faecalis, have been reported to regulate host energy production pathways, presumably to increase levels of 
metabolites required for their own growth [52-54]. However, acs-2 depletion had no effect on nematode lifespan upon bacterial challenge, suggesting that acs-2-dependent metabolites may not be limiting to E. faecalis growth. Consistent with this, depletion of the acs-2 negative regulator fat-7 also did not impact lifespan upon E. faecalis challenge, despite a marked increase in acs-2 induction. These data suggest that the protective effect of $n h r-49$ may be mediated through one or more of its other transcriptional targets [35], which may have as-yet uncharacterized roles in innate immunity. Alternatively, analogous to what has been reported for PPAR $\gamma$ and RelA [50], this effect could also be mediated through a physical interaction between NHR-49 and other nuclear hormone receptor family members [55]. Finally, although the vast majority of $n h r-49$ transcriptional targets play roles in fat metabolism $[35,56,57]$, the possibility of OG1RF sensitivity being mediated by targets that affect other biological processes remains open.

Depletion of $d l g-1$ or ajm-1 also resulted in reduced worm lifespan upon E. faecalis challenge. The DLG-1 / AJM-1 complex is a critical component of the C. elegans apical epithelial junction, which, as in other organisms, mediates cellular permeability, adhesion between neighboring cells, and cell polarity (reviewed in [39]). Loss of either protein results in developmental arrest at the embryonic elongation stage [58, 59]: Loss of $d l g-1$, which encodes a Membrane Associated GUanylate Kinase (MAGUK) protein family member, results in disappearance of the electron-dense apical junction structure in epidermal and intestinal epithelia, as well as mislocalization of AJM-1 [58]. Loss of ajm-1, encoding a novel protein with a large coiled-coil domain, produces bubble-like separations at cell-cell junctions that are normally in close apposition [59]. Here, we show that depletion of $d l g-1$ or $a j m-1$ from the L1 stage onwards affects the susceptibility of adult worms to bacteria, suggesting that epithelial junction integrity constitutes an important gut defense mechanism.

Although the role of DLG-1 / AJM-1 in apical junction integrity is well-characterized, a link between this and intestinal permeability remains to be demonstrated. This may be mediated through the claudin CLC-1, which is thought to interact with DLG-1 / AJM-1 at the cell surface-CLC-1deficient nematodes show compromised barrier function in the pharyngeal portion of the intestine, and leak ingested dye into the body cavity [60]. We hypothesize, however, that increased sensitivity to bacteria-derived toxins or small molecules (rather than dissemination of entire bacterial cells beyond the intestine) may be sufficient to bring about increased pathogen susceptibility.

The role of $d l g-1$ in the maintenance of the intestinal epithelial immune barrier appears to be conserved in Drosophila, where depletion of its fly homolog discs large $(d l g)$, a key component of the fly epithelial junction, results in over-proliferation of intestinal cells upon Pseudomonas aeruginosa infection [61]. Loss of other Drosophila epithelial junction components has also been reported to compromise gut defenses; notably, loss of the big bang (bbg) gene, which encodes a membrane-associated PDZ (PSD95, Discs-large, ZO-1) domain-containing protein, loosens epithelial junctions, allowing intestinal bacteria to constitutively activate fly midgut immune responses. The resulting chronic epithelial inflammation reduces lifespan [62].

While not fully understood, human IBD is also thought to stem from a breach in the intestinal epithelium, which exposes immune cells in the underlying lamina propria to bacteria or bacterial products from the gut (reviewed in [63]). Genetic variants in DLG5 (a human homolog of $C$. elegans dlg-1 and Drosophila dlg) are associated with IBD $[64,65]$, and intestinal permeability has been correlated with peripheral immune activation and clinical relapse in patients with Crohn's disease $[66,67]$. In IL-10 knockout mouse models of IBD, an unchecked $\mathrm{T}_{\mathrm{H}} 1$ inflammatory response is associated with increased intestinal permeability [68]; other mouse models indicate that disruption of intestinal epithelial junction integrity alone, through expression of dominant negative E-cadherin, is sufficient to initiate the disease [69]. In addition, consistent with the concept of gut bacteria-initiated systemic inflammation, increased intestinal permeability has been associated with metabolic conditions such as obesity, increased visceral adiposity, and fatty liver, both in patients and in animal models [70, 71].

\section{Conclusions}

We identified protective roles for $n h r-49, d l g-1$, and ajm-1 in $C$. elegans defense against the human gut commensal $E$. faecalis. The involvement of these genes suggests intriguing interactions between gut bacteria and the host processes of fatty acid metabolism, innate immunity, and epithelial junction integrity that consequently impact nematode survival. These data lend mechanistic support to the concept of gut microbiota dysbiosis in humans, where remarkably similar interactions have been proposed as a basis for the pathogenesis of inflammatory and metabolic diseases.

\section{Methods}

\section{Nematode and bacteria strains}

The C. elegans strain SS104 glp-4(bn2)I, a temperaturesensitive mutant that grows permissively at $15{ }^{\circ} \mathrm{C}$ but produces no progeny at $25^{\circ} \mathrm{C}$ [72], was used in survival assays. This was maintained at $15{ }^{\circ} \mathrm{C}$ on nematode growth media (NGM) agar and fed on the OP50 Escherichia coli strain, as described previously [73].

E. coli OP50 was grown at $37{ }^{\circ} \mathrm{C}$ in Luria-Bertani (LB) broth. OG1RF (ATCC accession number 47077), an Enterococcus faecalis strain of human origin, was grown in 
brain-heart infusion (BHI) broth (BD) at $37^{\circ} \mathrm{C}$. SGG53, a Streptococcus gallolyticus gallolyticus clinical isolate, was grown at $37{ }^{\circ} \mathrm{C}$ in tryptic soy (TS) broth (BD). mc(2)155 (ATCC accession number 700084), a Mycobacterium smegmatis strain, was grown at $37{ }^{\circ} \mathrm{C}$ in Middlebrook $7 \mathrm{H} 9$ broth supplemented with ADC enrichment (BD).

\section{RNAi knockdown of candidate genes}

Nematodes were fed on HT115 (DE3) E. coli clones transformed with either the empty L4440 vector (control), or the L4440 vector containing a gene-specific amplicon. RNAi constructs were either obtained from the Ahringer RNAi library [74] or created by cloning gene-specific amplicons into L4440 vector. Sequences of primers used for PCR amplification are listed in Additional file 2: Table S2.

Each clone was grown for $8 \mathrm{~h}$ in LB broth supplemented with $50 \mu \mathrm{g} / \mathrm{ml}$ ampicillin, seeded on NGM agar plates supplemented with $25 \mu \mathrm{g} / \mathrm{ml}$ carbenicillin and $1 \mathrm{mM} \beta$-D-1thiogalactopyranoside (IPTG), and left to induce overnight at room temperature. Nematode embryos, generated by hypochlorite treatment, were grown on these plates until the L4 stage, and subsequently transferred to pathogen lawns.

\section{Survival assays}

Pathogens were grown overnight in their respective liquid media at $37{ }^{\circ} \mathrm{C}$, seeded onto their respective agar plates, and left to form lawns overnight at $37{ }^{\circ} \mathrm{C}$. To minimize transfer of HT115 E. coli, L4-stage nematodes were allowed to crawl on bare agar before they were transferred to pathogen lawns. Subsequently, nematodes were transferred to fresh pathogen lawns every other day to further prevent growth of residual HT115 E. coli; none was visually observed. Nematode survival was monitored daily; nematodes were considered dead if they failed to respond to gentle touch with a platinum wire. The data presented are a pool of three independent experiments, with 20-30 nematodes each. Survival assays for OP50 and for each bacterial pathogen were performed on separate occasions; however, statistical comparisons presented in the Figures were made only within the same assay.

\section{Quantitative real-time PCR analysis}

Nematodes were collected in $1 \mathrm{ml}$ Trizol, and lysed by freeze-thawing. RNA was extracted from lysates with the Qiagen RNeasy Mini Kit (Qiagen), DNAse-treated (ThermoScientific), and used for cDNA synthesis with the Maxima H Minus First Strand cDNA Synthesis Kit (ThermoScientific). qRT-PCR was performed with the KAPA SYBR FAST qPCR Master Mix (KAPA Biosciences) on the LightCycler 480 II real-time thermocycler (Roche Applied Science). Raw values were normalized to each of three control genes (act-1, nhr-23, and ama$1)$; the average of the normalized values was then used for analysis [75]. Primer sequences used in qRT-PCR are presented in Additional file 3: Table S3.

\section{Statistical analysis}

Kaplan-Meier survival curve analysis and student t-tests were carried out with GraphPad Prism 3.

\section{Additional files}

Additional file 1: Table S1. C. elegans candidate genes screened via RNAi knockdown. IBD, inflammatory bowel disease; T2D, type 2 diabetes; KD, Kawasaki Disease. (DOCX 15 kb)

Additional file 2: Table S2. Sequences of primers used for PCR amplification of gene-specific amplicons for cloning into the $L 4440$ RNAi vector. RNAi constructs for candidate genes not listed here were obtained from the Ahringer RNAi library [74]. (DOCX $12 \mathrm{~kb}$ )

Additional file 3: Table S3. Sequences of primers used for quantitative RT-PCR. (DOCX $12 \mathrm{~kb}$ )

\section{Competing interests}

The authors declare that they have no competing interests.

\section{Authors' contributions}

SS designed and performed the experiments, analyzed the data, and wrote the manuscript. MLH conceived of the study and helped with experimental design, data analysis, and manuscript writing. Both authors read and approved the final manuscript.

\section{Acknowledgments}

This study was supported by the Agency for Science, Technology, and Research (A*STAR), Singapore. We thank Xiaohui Sem (Singapore Immunology Network) and Patrick Tan (Genome Institute of Singapore) for C. elegans strains and materials, and help with experimental techniques. We are grateful to Jan Gruber and Lifang Ng (Yale-National University of Singapore) for help with experimental techniques. We also thank Shugui Wang and Sven Pettersson (National Cancer Center) for providing the SGG53 S. gallolyticus gallolyticus strain.

\section{Author details}

${ }^{1}$ Infectious Diseases, Genome Institute of Singapore, 60 Biopolis Street, \#02-01 Genome, Singapore 138672, Singapore. ${ }^{2}$ Faculty of Infectious and Tropical Diseases, London School of Hygiene and Tropical Medicine, Keppel Street, London WC1E 7HT, United Kingdom.

Received: 2 September 2015 Accepted: 8 January 2016 Published online: 15 January 2016

\section{References}

1. Qin J, Li R, Raes J, Arumugam M, Burgdorf KS, Manichanh C, et al. A human gut microbial gene catalogue established by metagenomic sequencing. Nature. 2010;464:59-65.

2. Zhang Y-J, Li S, Gan R-Y, Zhou T, Xu D-P, Li H-B. Impacts of gut bacteria on human health and diseases. Int J Mol Sci. 2015;16:7493-519.

3. Ivanov II, Honda K. Intestinal commensal microbes as immune modulators. Cell Host Microbe. 2012;12:496-508.

4. Cadwell K, Patel KK, Maloney NS, Liu T-C, Ng ACY, Storer CE, et al. Virus-plussusceptibility gene interaction determines Crohn's disease gene Atg16L1 phenotypes in intestine. Cell. 2010;141:1135-45.

5. Björkholm B, Bok CM, Lundin A, Rafter J, Hibberd ML, Pettersson S. Intestinal microbiota regulate xenobiotic metabolism in the liver. PLoS One. 2009;4, e6958.

6. Heijtz RD, Wang S, Anuar F, Qian Y, Björkholm B, Samuelsson A, et al. Normal gut microbiota modulates brain development and behavior. Proc Natl Acad Sci. 2011;108:3047-52.

7. Lundin A, Bok CM, Aronsson L, Björkholm B, Gustafsson J-Å, Pott S, et al. Gut flora, Toll-like receptors and nuclear receptors: a tripartite communication that tunes innate immunity in large intestine. Cell Microbiol. 2008;10:1093-103. 
8. Bäckhed F, Ding H, Wang T, Hooper LV, Koh GY, Nagy A, et al. The gut microbiota as an environmental factor that regulates fat storage. Proc Nat Acad Sci U S A. 2004;101:15718-23.

9. Bäckhed F, Fraser CM, Ringel Y, Sanders ME, Sartor RB, Sherman PM, et al. Defining a healthy human gut microbiome: current concepts, future directions, and clinical applications. Cell Host Microbe. 2012;12:611-22.

10. Dalal SR, Chang EB. The microbial basis of inflammatory bowel diseases. J Clin Invest. 2014;124:4190-6.

11. Musso G, Gambino R, Cassader M. Obesity, diabetes, and gut microbiota the hygiene hypothesis expanded? Diabetes Care. 2010;33:2277-84.

12. Willing BP, Dicksved J, Halfvarson J, Andersson AF, Lucio M, Zheng Z, et al. A pyrosequencing study in twins shows that gastrointestinal microbial profiles vary with inflammatory bowel disease phenotypes. Gastroenterology. 2010;139:1844-54. e1.

13. Lupp C, Robertson ML, Wickham ME, Sekirov I, Champion OL, Gaynor EC, et al. Host-mediated inflammation disrupts the intestinal microbiota and promotes the overgrowth of enterobacteriaceae. Cell Host Microbe. 2007;2:119-29

14. Morgan XC, Tickle TL, Sokol H, Gevers D, Devaney KL, Ward DV, et al. Dysfunction of the intestinal microbiome in inflammatory bowel disease and treatment. Genome Biol. 2012:13:R79.

15. Sokol H, Pigneur B, Watterlot L, Lakhdari O, Bermúdez-Humarán LG, Gratadoux J-J, et al. Faecalibacterium prausnitzii is an anti-inflammatory commensal bacterium identified by gut microbiota analysis of Crohn disease patients. Proc Natl Acad Sci. 2008;105:16731-6.

16. Qin J, Li Y, Cai Z, Li S, Zhu J, Zhang F, et al. A metagenome-wide association study of gut microbiota in type 2 diabetes. Nature. 2012;490:55-60.

17. Turnbaugh PJ, Ley RE, Mahowald MA, Magrini V, Mardis ER, Gordon Jl. An obesity-associated gut microbiome with increased capacity for energy harvest. Nature. 2006:444:1027-131.

18. Ley RE, Turnbaugh PJ, Klein S, Gordon Jl. Microbial ecology: human gut microbes associated with obesity. Nature. 2006:444:1022-3.

19. Turnbaugh PJ, Hamady M, Yatsunenko T, Cantarel BL, Duncan A, Ley RE, et al. A core gut microbiome in obese and lean twins. Nature. 2009:457:480-4.

20. Khor CC, Hibberd ML. Host-pathogen interactions revealed by human genome-wide surveys. Trends Genet. 2012;28:233-43.

21. Anderson CA, Boucher G, Lees CW, Franke A, D'Amato M, Taylor KD, et al. Meta-analysis identifies 29 additional ulcerative colitis risk loci, increasing the number of confirmed associations to 47. Nat Genet. 2011;43:246-52.

22. Franke A, McGovern DPB, Barrett JC, Wang K, Radford-Smith GL, Ahmad T, et al. Genome-wide meta-analysis increases to 71 the number of confirmed Crohn's disease susceptibility loci. Nat Genet. 2010;42:1118-25.

23. McGovern DPB, Gardet A, Törkvist L, Goyette P, Essers J, Taylor KD, et al. Genome-wide association identifies multiple ulcerative colitis susceptibility loci. Nat Genet. 2010;42:332-7.

24. Scott LJ, Mohlke KL, Bonnycastle LL, Willer CJ, Li Y, Duren WL, et al. A genome-wide association study of type 2 diabetes in Finns detects multiple susceptibility variants. Science. 2007;316:1341-5.

25. Burton PR, Clayton DG, Cardon LR, Craddock N, Deloukas P, Duncanson A, et al. Genome-wide association study of 14,000 cases of seven common diseases and 3,000 shared controls. Nature. 2007:447:661-78.

26. Inohara N, Ogura Y, Fontalba A, Gutierrez O, Pons F, Crespo J, et al. Host recognition of bacterial Muramyl Dipeptide Mediated through NOD2 IMPLICATIONS FOR CROHN'S DISEASE. J Biol Chem. 2003;278:5509-12.

27. Wong SH, Gochhait S, Malhotra D, Pettersson FH, Teo YY, Khor CC, et al. Leprosy and the adaptation of human toll-like receptor 1. PLoS Pathog. 2010;6, e1000979.

28. Zhang F, Liu H, Chen S, Low H, Sun L, Cui Y. Identification of two new loci at IL23R and RAB32 that influence susceptibility to leprosy. Nat Genet. 2011:43:1247-51.

29. Zhang F-R, Huang W, Chen S-M, Sun L-D, Liu H, Li Y, et al. Genomewide association study of leprosy. N Engl J Med. 2009;361:2609-18.

30. Sem X, Rhen M. Pathogenicity of salmonella enterica in Caenorhabditis elegans Relies on disseminated oxidative stress in the infected host. PLoS One. 2012;7, e45417

31. Garsin DA, Sifri CD, Mylonakis E, Qin X, Singh KV, Murray BE, et al. A simple model host for identifying Gram-positive virulence factors. Proc Natl Acad Sci. 2001;98:10892-7.

32. Bolm M, Jansen WTM, Schnabel R, Chhatwal GS. Hydrogen peroxidemediated killing of Caenorhabditis elegans: a common feature of different streptococcal species. Infect Immun. 2004;72:1192-4.
33. Kirienko NV, Kirienko DR, Larkins-Ford J, Wählby C, Ruvkun G, Ausubel FM. Pseudomonas aeruginosa disrupts caenorhabditis elegans iron homeostasis, causing a hypoxic response and death. Cell Host Microbe. 2013;13:406-16.

34. Palmer KL, Kos VN, Gilmore MS. Horizontal gene transfer and the genomics of enterococcal antibiotic resistance. Curr Opin Microbiol. 2010;13:632-9. Antimicrobials/Genomics.

35. Gilst MRV, Hadjivassiliou H, Jolly A, Yamamoto KR. Nuclear hormone receptor nhr-49 controls fat consumption and fatty acid composition in C. elegans. PLoS Biol. 2005;3:e53.

36. Collins D, Hogan AM, Winter DC. Microbial and viral pathogens in colorectal cancer. Lancet Oncol. 2011;12:504-12.

37. Boleij A, Muytjens CMJ, Bukhari SI, Cayet N, Glaser P, Hermans PWM, et al. Novel clues on the specific association of streptococcus gallolyticus subsp gallolyticus with colorectal cancer. J Infect Dis. 2011;203:1101-9.

38. Packey CD, Sartor RB. Commensal bacteria, traditional and opportunistic pathogens, dysbiosis and bacterial killing in inflammatory bowel diseases. Curr Opin Infect Dis. 2009;22:292-301.

39. Lynch AM, Hardin J. The assembly and maintenance of epithelial junctions in C. elegans. Front Biosci J Virtual Libr. 2009:14:1414-32.

40. Gupta RK, Kaestner KH. HNF-4a: from MODY to late-onset type 2 diabetes. Trends Mol Med. 2004:10:521-4.

41. Wahli W, Michalik L. PPARs at the crossroads of lipid signaling and inflammation. Trends Endocrinol Metab. 2012;23:351-63. Special Focus: Mammalian Circadian Rhythms and Metabolism.

42. Larsen TM, Toubro S, Astrup A. PPARgamma agonists in the treatment of type II diabetes: is increased fatness commensurate with long-term efficacy? Int J Obes. 2003;27:147-61.

43. Annese V, Rogai F, Settesoldi A, Bagnoli S. PPARgamma in inflammatory bowel disease. PPAR Res. 2012;2012, e620839.

44. Wilding JPH. PPAR agonists for the treatment of cardiovascular disease in patients with diabetes. Diabetes Obes Metab. 2012;14:973-82.

45. Cani PD, Amar J, Iglesias MA, Poggi M, Knauf C, Bastelica D, et al. Metabolic endotoxemia initiates obesity and insulin resistance. Diabetes. 2007:56:1761-72

46. Cani PD, Bibiloni R, Knauf C, Waget A, Neyrinck AM, Delzenne NM, et al. Changes in gut microbiota control metabolic endotoxemia-induced inflammation in high-fat diet-induced obesity and diabetes in mice. Diabetes. 2008:57:1470-81.

47. Odegaard Jl, Ricardo-Gonzalez RR, Goforth MH, Morel CR, Subramanian V, Mukundan L, et al. Macrophage-specific PPARy controls alternative activation and improves insulin resistance. Nature. 2007;447:1116-20.

48. Odegaard JI, Ricardo-Gonzalez RR, Eagle AR, Vats D, Morel CR, Goforth MH, et al. Alternative (M2) activation of Kupffer cells by PPAR ameliorates obesity-induced insulin resistance. Cell Metab. 2008;7:496-507.

49. Coll T, Álvarez-Guardia D, Barroso E, Gómez-Foix AM, Palomer X, Laguna JC, et al. Activation of peroxisome proliferator-activated receptor- $\delta$ by GW501516 prevents fatty acid-induced nuclear factor-kB activation and insulin resistance in skeletal muscle cells. Endocrinology. 2010;151:1560-9.

50. Kelly D, Campbell Jl, King TP, Grant G, Jansson EA, Coutts AGP, et al. Commensal anaerobic gut bacteria attenuate inflammation by regulating nuclear-cytoplasmic shuttling of PPAR-y and RelA. Nat Immunol. 2004;5:104-12.

51. Are A, Aronsson L, Wang S, Greicius G, Lee YK, Gustafsson J-Å, et al. Enterococcus faecalis from newborn babies regulate endogenous PPARY activity and IL-10 levels in colonic epithelial cells. Proc Natl Acad Sci. 2008;105:1943-8.

52. Wong HEE, Li M-S, Kroll JS, Hibberd ML, Langford PR. Genome wide expression profiling reveals suppression of host defence responses during Colonisation by Neisseria meningitides but not N. lactamica. PLoS One. 2011:6:e26130.

53. Hooper LV, Xu J, Falk PG, Midtvedt T, Gordon Jl. A molecular sensor that allows a gut commensal to control its nutrient foundation in a competitive ecosystem. Proc Natl Acad Sci. 1999;96:9833-8.

54. Lindenstrauß AG, Ehrmann MA, Behr J, Landstorfer R, Haller D, Sartor RB, et al. Transcriptome analysis of enterococcus faecalis toward its adaption to surviving in the mouse intestinal tract. Arch Microbiol. 2014;196:423-33.

55. Li S, Armstrong CM, Bertin N, Ge H, Milstein S, Boxem M, et al. A map of the interactome network of the Metazoan C elegans. Science. 2004:303:540-3.

56. Gilst MRV, Hadjivassiliou H, Yamamoto KR. A Caenorhabditis elegans nutrient response system partially dependent on nuclear receptor NHR-49. Proc Natl Acad Sci U S A. 2005;102:13496-501. 
57. Fredens J, Færgeman NJ. Quantitative proteomics by amino acid labeling identifies novel NHR-49 regulated proteins in C. elegans. Worm. 2012;1:66-71.

58. McMahon $L$, Legouis $R$, Vonesch $J$ - $L$, Labouesse $M$. Assembly of $C$. elegans apical junctions involves positioning and compaction by LET413 and protein aggregation by the MAGUK protein DLG-1. J Cell Sci. 2001;114:2265-77

59. Köppen M, Simske JS, Sims PA, Firestein BL, Hall DH, Radice AD, et al. Cooperative regulation of AJM-1 controls junctional integrity in Caenorhabditis elegans epithelia. Nat Cell Biol. 2001;3:983-91.

60. Asano A, Asano K, Sasaki H, Furuse M, Tsukita S. Claudins in caenorhabditis elegans: their distribution and barrier function in the epithelium. Curr Biol. 2003;13:1042-6.

61. Apidianakis Y, Pitsouli C, Perrimon N, Rahme L. Synergy between bacterial infection and genetic predisposition in intestinal dysplasia. Proc Natl Acad Sci. 2009;106:20883-8.

62. Bonnay F, Cohen-Berros E, Hoffmann M, Kim SY, Boulianne GL, Hoffmann $J A$, et al. big bang gene modulates gut immune tolerance in Drosophila. Proc Natl Acad Sci U S A. 2013;110:2957-62.

63. Clayburgh DR, Shen L, Turner JR. A porous defense: the leaky epithelial barrier in intestinal disease. Lab Invest. 2004;84:282-91.

64. Stoll M, Corneliussen B, Costello CM, Waetzig GH, Mellgard B, Koch WA, et al. Genetic variation in DLG5 is associated with inflammatory bowel disease. Nat Genet. 2004;36:476-80

65. Büning C, Geerdts L, Fiedler T, Gentz E, Pitre G, Reuter W, et al. DLG5 variants in inflammatory bowel disease. Am J Gastroenterol. 2006;101:786-92.

66. Wyatt J, Vogelsang H, Hübl W, Waldhöer T, Lochs H. Intestinal permeability and the prediction of relapse in Crohn's disease. Lancet. 1993;341:1437-9.

67. Yacyshyn BR, Meddings JB. CD45RO expression on circulating CD19+ B cells in Crohn's disease correlates with intestinal permeability. Gastroenterology. 1995;108:132-7.

68. Davidson NJ, Leach MW, Fort MM, Thompson-Snipes L, Kühn R, Müller W, et al. T helper cell 1-type CD4+ T cells, but not B cells, mediate colitis in interleukin 10-deficient mice. J Exp Med. 1996;184:241-51.

69. Hermiston ML, Gordon JI. Inflammatory bowel disease and adenomas in mice expressing a dominant negative N-cadherin. Science. 1995;270:1203-7.

70. Gummesson A, Carlsson LMS, Storlien LH, Bäckhed F, Lundin P, Löfgren L, et al. Intestinal permeability is associated with visceral adiposity in healthy women. Obesity. 2011:19:2280-2.

71. Brun P, Castagliuolo I, Leo VD, Buda A, Pinzani M, Palù G, et al. Increased intestinal permeability in obese mice: new evidence in the pathogenesis of nonalcoholic steatohepatitis. Am J Physiol - Gastrointest Liver Physiol. 2007;292:G518-25.

72. Beanan MJ, Strome S. Characterization of a germ-line proliferation mutation in C. elegans. Development. 1992;116:755-66.

73. Brenner S. The genetics of Caenorhabditis Elegans. Genetics. 1974;77:71-94.

74. Kamath RS, Fraser AG, Dong Y, Poulin G, Durbin R, Gotta M, et al. Systematic functional analysis of the Caenorhabditis elegans genome using RNAi. Nature. 2003;421:231-7.

75. Gaglia MM, Jeong D-E, Ryu E-A, Lee D, Kenyon C, Lee S-J. Genes that act downstream of sensory neurons to influence Longevity, Dauer formation, and pathogen responses in Caenorhabditis elegans. PLoS Genet. 2012;8, e1003133.

\section{Submit your next manuscript to BioMed Central and we will help you at every step:}

- We accept pre-submission inquiries

- Our selector tool helps you to find the most relevant journal

- We provide round the clock customer support

- Convenient online submission

- Thorough peer review

- Inclusion in PubMed and all major indexing services

- Maximum visibility for your research

Submit your manuscript at www.biomedcentral.com/submit

) Biomed Central 